\title{
NONLINEAR KINETICS OF SPINODAL DECOMPOSITION, AND DISSOLUTION OF INHOMOGENEITIES FORMED BY SPINODAL DECOMPOSITION IN POLYMER BLENDS
}

\author{
A. Ziya Akcasu* \\ Department of Nuclear Engineering, The University of Michigan, Ann Arbor, \\ Michigan 48109 \\ B. Erman and I. Bahar \\ Polymer Research Center and School of Engineering, Bogazici University, Bebek \\ 80815 , Istanbul, Turkey
}

\begin{abstract}
Nonlinear kinetics of both spinodal decomposition at early stages, and the dissolution of homogeneities formed during spinodal decomposition, is studied. Variation of the scattering intensity during a complete cycle consisting of a step temperature change from $T_{1}$ in the one-phase region to $T_{2}$ in the two-phase region, a period of spinodal decomposition followed by a temperature drop from $T_{2}$ back to $T_{1}$, and the subsequent relaxation to the original equilibrium state, is investigated at various wavenumbers. Step temperature changes within one-phase region are also investigated.
\end{abstract}

\section{INTRODUCTION}

In this paper we investigate the kinetics of dissolution and demixing processes taking into account the nonlinearities and the effect of thermal fluctuations. The theoretical approach taken in this study is similar in principle to that by Langer et al., originally developed for fluid mixtures (Ref.1,2). There are, however, differences in the way the fluctuations are included in the theory, and in the details of the derivations arising from the chain connectivity (polymer effect) in the case of polymer blends. In addition, the variation of the scattering intensity as a function of time at various wavenumbers is studied numerically during a complete cycle consisting of a step temperature change from $T_{1}$ in the one-phase region to $T_{2}$ in the twophase region, a period of spinodal decomposition followed by a temperature drop from $T_{2}$ back to $T_{1}$, and the subsequent relaxation to the original equilibrium state. The transients in the intensity following step temperature changes within one-phase region are also analyzed. The present study was motivated by the the recent light and neutron scattering experiments on the polymer blend of polystyrene and poly(vinyl methyl ether) by Han and his coworkers (Ref.3,4), involving spinodal decomposition (demixing), and dissolution of homogeneities formed during the spinodal decomposition following a temperature drop into the one phase region. The comparison of the theoretical predictions obtained in this work and the experimental results will be reported in a future publication. 


\section{DESCRIPTION OF THE THEORY}

We consider a melt of two homopolymer species $\mathrm{A}$ and $\mathrm{B}$. The local volume fractions of monomers at a point $\mathbf{r}$ and time $t$ are denoted by $\phi_{A}(r, t)$ and $\phi_{B}(r, t)$. The mixture is assumed to be incompressible so that $\phi_{A}(r, t)+\phi_{B}(r, t)=1$. When the mixture is in an homogeneous equilibrium state, the volume fractions are uniform and denoted by $\phi_{A}=\phi_{O}$ and $\phi_{B}=1-\phi_{O}$. The incremental volume fraction of the A component is defined as $\varphi(r, t) \equiv \phi_{A}(r, t)-\phi_{0}$. The monomeric volumes $\mathrm{v}_{\mathrm{A}}$ and $\mathrm{v}_{\mathrm{B}}$ of the species are allowed to be different. We assume that the Amonomers are labelled.

The intensity $\mathbf{I}(\mathrm{q}, \mathrm{t})$ of the scattering beam in a scattering experiment is related to the Fourier transform of $\varphi(\mathbf{r}, \mathbf{l})$ :

$\left.I(q, t)=\frac{V}{v_{0}}<\left|\varphi_{q}(t)\right|^{2}\right\rangle$

where $\mathbf{q}$ is the momentum transfer vector, $\mathrm{v}_{0}$ is a reference volume to be specified later, and $\varphi_{\mathbf{q}}(\mathrm{t})$ denotes the discrete Fourier transform of $\varphi(\mathbf{r}, \mathrm{t})$, i.e.,

$\varphi_{\mathbf{q}}(\mathrm{t})=\frac{1}{V} \int_{V} \mathrm{~d}^{3} \mathrm{re}^{\mathrm{i} \mathbf{q} \cdot \mathbf{r}} \varphi(\mathbf{r}, \mathrm{l})$

in which $V$ is the volume of the system. The $\varphi(r, t)$ and $\varphi_{\mathbf{q}}(t)$ are both dimensionless. The factor $\mathrm{V} / \mathrm{v}_{\mathrm{o}}$ in Eq. 1 is introduced only for notational simplicity in the following derivations. When the mixture is at equilibrium, $\mathrm{I}(\mathrm{q}, \mathrm{t})$ is independent of time, and is given by $\mathrm{I}_{\mathrm{eq}}(\mathrm{q})=\mathrm{S}_{\mathrm{AA}}(\mathrm{q})$, where $S_{A A}(q)$ is the static structure factor calculated by de Gennes (Ref.5) using the random phase approximation, as

$\frac{1}{S_{A A}(q)}=\frac{1}{z_{A} N_{A} \phi_{0} f_{D}\left(q^{2} R_{g A}^{2}\right)}+\frac{1}{z_{B} N_{B}\left(1-\phi_{0}\right) f_{D}\left(q^{2} R_{g B}^{2}\right)}-2 \chi$

in which $N_{\alpha}$ and $R_{g \alpha}$ denote, respectively, the number of monomers in, and the radius of gyration of, a chain of kind $\alpha=A, B$, and $f_{D}(x)$ is the usual Debye function

$\mathrm{f}_{\mathrm{D}}(\mathrm{x})=\frac{2}{\mathrm{x}^{2}}\left(\mathrm{x}-1+\mathrm{e}^{-\mathrm{x}}\right)$

In Eq.2, $\chi$ is the Flory interaction parameter, and $\mathrm{z}_{\alpha}=\mathrm{v}_{\alpha /} / \mathrm{v}_{\mathrm{O}}$ denotes the normalized monomeric volumes relative to the reference volume $v_{0}$. The $\mathrm{I}(q, \mathrm{l})$ becomes time dependent during transients following, for example, a temperature jump from an initial temperature $T_{i}$ in the one-phase region to a final temperature $\mathrm{T}_{\mathrm{f}}$ in the spinodal region, leading to spinodal decomposition (demixing), or a temperature drop from $T_{i}$ in the two-phase region to $T_{f}$ in the one-phase region (reverse quench experiment involving dissolution), or a step temperature change from $T_{i}$ to $T_{f}<T_{i}$, or vice versa, within the one-phase region. 
The purpose of this paper is to analytically investigate the time dependence of the intensity $\mathbf{I}(\mathrm{q}, \mathrm{t})$ as a function of $\mathrm{q}$ and $\mathrm{t}$ during both demixing and dissolution, as well as during transients following step temperature changes within one-phase region, in order to interprete the recent experiments by Han at NIST (Ref.3,4).

Following Binder (Ref.6), we start with the free energy excess for a binary mixture of A and B homopolymers:

$$
\frac{\Delta F}{\mathrm{k}_{\mathrm{B}} \mathrm{T}}=\int \mathrm{d}^{3} \mathrm{r} \frac{1}{\mathrm{v}_{\mathrm{o}}}\left\{\mathrm{f}[\phi(\mathbf{r})]+\frac{1}{36}\left[\frac{\sigma_{\mathrm{A}}^{2}}{\mathrm{z}_{\mathrm{A}} \phi(\mathbf{r})}+\frac{\sigma_{\mathrm{B}}^{2}}{\mathrm{z}_{\mathrm{B}}[1-\phi(\mathbf{r})]}\right]|\nabla \phi(\mathbf{r})|^{2}\right\}
$$

where $\phi(\mathbf{r})=\phi_{\mathrm{A}}(\mathbf{r}), \mathrm{k}_{\mathrm{B}} \mathrm{T}$ is the temperature in energy units, and

$$
f(\phi)=\frac{\phi}{z_{A} N_{A}} \ln \phi+\frac{(1-\phi)}{z_{B} N_{B}} \ln (1-\phi)+\chi \phi(1-\phi)
$$

In Eq.4a, $\sigma_{\alpha}$ denotes the statistical segment lengths of an $\alpha$-chain. When $\mathrm{v}_{\mathrm{A}}=\mathrm{v}_{\mathrm{B}}=\mathrm{v}_{\mathbf{O}}$, and $\mathrm{v}_{\mathrm{O}}$ is set equal to unity, Eqs.4 reduces to Eq.2.1 and 2.2 of Binder (Ref.6) (we will use his notations as much as possible to ease comparison). Eq.4 was previously given, in the case of $v_{A} \neq v_{B}$, by Shibayama, et al., without the gradient term (Ref.7), in which the reference volume $v_{O}$ was chosen as $v_{O}=\sqrt{v_{A} v_{B}}$. Although the choice of $v_{O}$ is arbitrary, this particular one has the advantage that it leads to $\mathrm{z}_{\mathrm{A}} \mathrm{z}_{\mathrm{B}}=1$, and thus makes the quantity in the curly bracket in Eq.4a a function of the ratio of the segmental volumes only. The coefficient of $|\nabla \phi(\mathbf{r})|^{2}$ in Eq.4a is chosen by de Gennes (Ref.8) in such a way that the above expression $\Delta F$ leads to the small-q limit of the static structure factor $S_{\mathrm{AA}}(q)$ in Eq.2:

$\frac{1}{S_{A A}(q)}=2\left\{\chi_{s}-\chi+\frac{q^{2}}{36}\left[\frac{\sigma_{A}^{2}}{z_{A} \phi_{0}}+\frac{\sigma_{B}^{2}}{z_{B}\left(1-\phi_{0}\right)}\right]\right\}$

where $\chi_{S}$ denotes the value of the interaction parameter on the spinodal, i. e.,

$\chi_{S}=\frac{1}{2}\left[\frac{1}{z_{A} N_{A} \phi_{0}}+\frac{1}{z_{B} N_{B}\left(1-\phi_{0}\right)}\right]$

which is obtained by expanding the Debye function as $f_{D}(x)=1-x / 3$ for small arguments. It is possible to modify the expression of the free energy, as shown by Akcasu and Sanchez (Ref.9), in such a way that it will reproduce the full expression of $S_{A A}(q)$ in Eq.2 for all wave numbers . This extension will not be needed in the interpretation of the step temperature change experiments we envisage, because the Debye function can be approximated by $f_{D}(x)=1-x / 3$ in the q-range involved in them.

The functional derivative of $\Delta F$ yields the local chemical potential difference (Ref.6) $\mu(\mathbf{r})$ : 


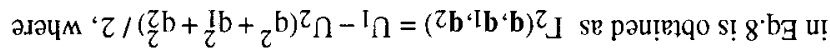

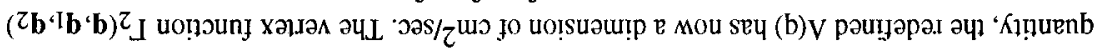

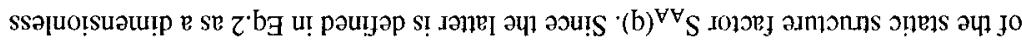

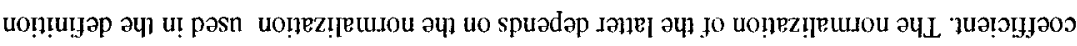
دวลิpsu $O$

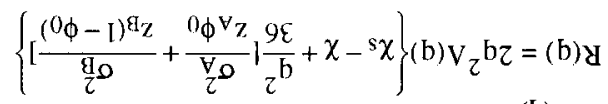

:(b) y jo

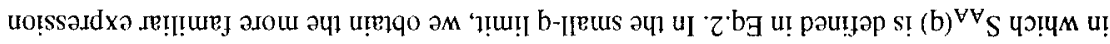

$$
\frac{(b)^{\vee v} S}{(b) V} z^{b}=(b) \&
$$

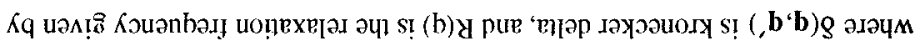

$$
\text { (1) } \mathbf{b} \mathrm{u}+
$$

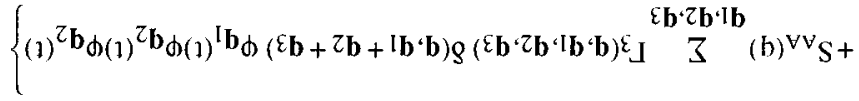

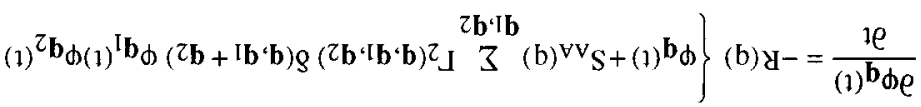

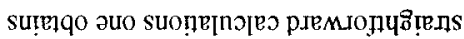

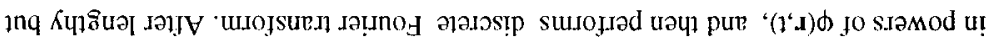

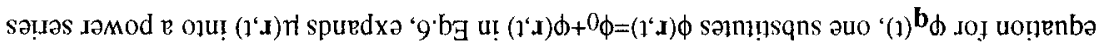

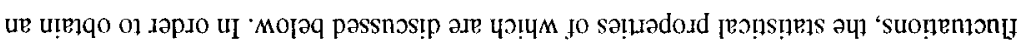

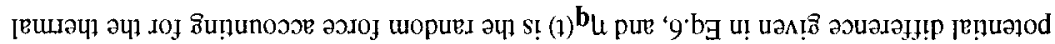

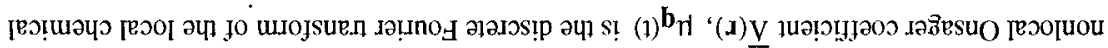

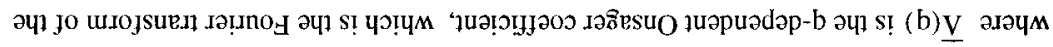

$$
\text { (1) } \mathbf{b}^{\mathbf{b}} \mathrm{u}+(1)^{\mathbf{b}_{1}} \frac{L^{\mathrm{I}} \mathrm{x}}{(\mathrm{b}) \underline{\mathrm{V}}} z^{\mathrm{b}-=}=\frac{1 e}{(1)^{\mathbf{b}_{d}} \mathrm{e}}
$$

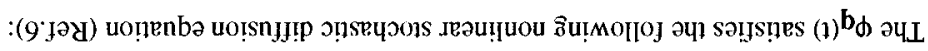

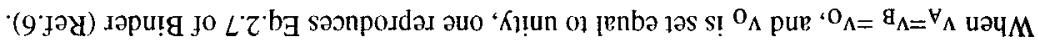

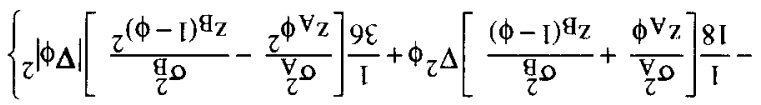

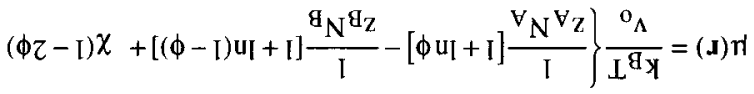


$\mathrm{U}_{1}=\frac{1}{2}\left[\frac{1}{\mathrm{z}_{\mathrm{B}} \mathrm{N}_{\mathrm{B}}\left(1-\phi_{0}\right)^{2}}-\frac{1}{\mathrm{z}_{\mathrm{A}} \mathrm{N}_{\mathrm{A}} \phi_{0}^{2}}\right]$

$\mathrm{U}_{2}=\frac{1}{36}\left[\frac{\sigma_{\mathrm{A}}^{2}}{\mathrm{z}_{\mathrm{A}} \phi_{\mathrm{O}}^{2}}-\frac{\sigma_{\mathrm{B}}^{2}}{\mathrm{z}_{\mathrm{B}}\left(1-\phi_{0}\right)^{2}}\right]$

In obtaining Eq. 11 , we symmetrized the second term in Eq.8 by interchanging $\mathbf{q}_{1}$ and $\mathbf{q}_{2}$, adding the resulting equations and dividing by 2 . It is to be noted that $\Gamma_{2}\left(\mathbf{q}, \mathbf{q}_{1}, \mathbf{q}_{2}\right)$ vanishes in the case of a symmetric mixture in which the molecules of the two species are identical to each other so that $\mathrm{N}_{\mathrm{A}}=\mathrm{N}_{\mathrm{B}}, \mathrm{z}_{\mathrm{A}}=\mathrm{z}_{\mathrm{B}}, \sigma_{\mathrm{A}}=\sigma_{\mathrm{B}}$, and $\mathrm{v}_{\mathrm{A}}=\mathrm{v}_{\mathrm{B}}$, and their volume fractions are equal, i.e., $\phi_{\mathrm{o}}=1 / 2$.

The cubic vertex function in Eq. 8 is $\Gamma_{3}\left(\mathbf{q}, \mathbf{q}_{1}, \mathbf{q}_{2}, \mathbf{q}_{3}\right)=z_{1}+z_{2}\left(q^{2}+q_{1}^{2}+q_{2}^{2}+q_{3}^{2}\right) / 6$ where

$\mathrm{Z}_{1}=\frac{1}{3}\left[\frac{1}{\mathrm{z}_{\mathrm{A}} \mathrm{N}_{\mathrm{A}} \phi_{\mathrm{O}}^{3}}+\frac{1}{\mathrm{z}_{\mathrm{B}} \mathrm{N}_{\mathrm{B}}\left(1-\phi_{\mathrm{O}}\right)^{3}}\right]$

$\mathrm{z}_{2}=\frac{1}{18}\left[\frac{\sigma_{\mathrm{A}}^{2}}{\mathrm{z}_{\mathrm{A}} \phi_{\mathrm{O}}^{3}}+\frac{\sigma_{\mathrm{B}}^{2}}{\mathrm{z}_{\mathrm{B}}\left(1-\phi_{0}\right)^{3}}\right]$

In Eq. 8 we terminated the expansion of $\mu(\mathbf{r}, \mathrm{t})$ in powers of $\varphi(r, t)$ after the cubic term.

We now come to the specification of the statistical properties of $\eta_{q}(t)$. It is assumed to be a delta correlated (white) random process with zero mean, and an autocovariance $\mathrm{C}(\mathrm{q})$, i.e.,

$<\eta_{\mathbf{q}}(\mathrm{t})>=0$

$<\eta_{\left.q^{(}\right)}(t) \eta_{-}\left(t^{\prime}\right)>=\delta\left(t-t^{\prime}\right) \bar{C}(q)$

$<\eta_{\mathbf{q}}(\mathrm{t}) \varphi_{\left.-\mathbf{q}^{(t)}\right)+<\varphi_{\mathbf{q}}}(\mathrm{t}) \eta_{-q^{(}}(\mathrm{t})>=\bar{C}(\mathrm{q})$

The origin of these properties, and the approximations inherent in them have been discused elsewhere (Ref.10).

The task now is to obtain an equation for the intensity $I(q, t)=\left(V / v_{0}\right)<\left|\varphi_{q^{(t)}}\right|^{2}>$ using the above stochastic nonlinear description of the mixture. For this purpose we multiply Eq. 8 by $\varphi_{-q}(t)$, and its complex conjugate by $\varphi_{q}(t)$, and add them up. We then take the ensemble average of the resulting equations, and make use of Eq.13b. The result reads

$$
\begin{aligned}
\frac{\mathrm{dI}(\mathrm{q}, \mathrm{t})}{\mathrm{dt}}=- & -2 \mathrm{R}(\mathrm{q})\left\{\mathrm{I}(\mathrm{q}, \mathrm{t})+\mathrm{S}_{\mathrm{AA}}(\mathrm{q}) \frac{\mathrm{V}}{\mathrm{v}_{\mathrm{o}}}\left[\sum_{\mathbf{q}_{1}, \mathbf{q}_{2}} \Gamma_{2}\left(\mathbf{q}, \mathbf{q}_{1}, \mathbf{q}_{2}\right) \delta\left(\mathbf{q}, \mathbf{q}_{1}+\mathbf{q}_{2}\right)\right.\right. \\
& <\varphi_{\mathbf{q}_{1}}(\mathrm{t}) \varphi_{\mathbf{q}_{2}}(\mathrm{t}) \varphi_{-\mathbf{q}}(\mathrm{t})>+\sum_{\mathbf{q}_{1}, \mathbf{q}_{2}, \mathbf{q}_{3}} \Gamma_{3}\left(\mathbf{q}, \mathbf{q}_{1}, \mathbf{q}_{2}, \mathbf{q}_{3}\right) \delta\left(\mathbf{q}, \mathbf{q}_{1}+\mathbf{q}_{2}+\mathbf{q}_{3}\right) \\
& \left.\left.<\varphi_{\mathbf{q}_{1}}(\mathrm{t}) \varphi_{\mathbf{q}_{2}}(\mathrm{t}) \varphi_{\mathbf{q}_{2}}(\mathrm{t}) \varphi_{-\mathbf{q}}(\mathrm{t})>\right]\right\}+\mathrm{C}(\mathrm{q})
\end{aligned}
$$


where we have redefined $C(q)=\left(V / v_{0}\right) \bar{C}(q)$. In the linear theory, Eq.14 is approximated by $\mathrm{dI}(\mathrm{q}, \mathrm{t}) / \mathrm{dt}=-2 \mathrm{R}(\mathrm{q}) \mathrm{I}(\mathrm{q}, \mathrm{t})+\mathrm{C}(\mathrm{q})$ with a solution

$I(q, t)=I_{i n}(q, t) e^{-2 R(q) t}+I_{e q}(q)\left[1-e^{-2 R(q) t}\right]$

which is the celebrated Cahn-Hilliard-Cook form ( $\mathrm{CHC}$ ) for polymer mixtures (Ref.11,12). In obtaining Eq.15, we have eliminated $\mathrm{C}(\mathrm{q})$ requiring that $\mathrm{I}(\mathrm{q}, \mathrm{t}) \rightarrow \mathrm{I}_{\mathrm{eq}}(\mathrm{q})$ as $\mathrm{t} \rightarrow \infty$. This leads to

$C(q)=2 R(q) I_{e q}(q)$

which is the Einstein's relation (Ref.13), or a statement of the fluctuation-dissipation theorem in linear systems. We note that this procedure is applicable when the final state is an equilibrium state, which is the case in reverse quench experiments, or those involving step temperature changes in one-phase region. We shall comment later on the choice of $\mathrm{C}(\mathrm{q})$ when the final temperature is in the spinodal region, i.e., when the final state is an unstable state. We mention in passing that in a recent publication (Ref.10) one of us provided a non-markovian extension of the $\mathrm{CHC}$ theory in matrix form using a microscopic approach based on the Zwanzig-Mori projection operator technique, and investigated the nature of approximations in Eq.15. In the special case of scattering from a one-component system, the extended derivation results in

$\mathbf{I}(\mathrm{q}, \mathrm{t})=\mathrm{I}_{\text {in }}(\mathrm{q}) S^{2}(\mathrm{q}, \mathrm{t})+\mathrm{I}_{\mathrm{eq}}(\mathrm{q})\left[1-S^{2}(\mathrm{q}, \mathrm{t})\right]$

where $S(\mathrm{q}, \mathrm{t})$ is the normalized $(S(\mathrm{q}, 0)=1)$ dynamic (or intermediate) scattering function in the final equilibrium state, i.e.,

$S(\mathrm{q}, \mathrm{t})=\frac{\left\langle\varphi_{\mathbf{q}}(\mathrm{t}) \varphi_{\mathbf{q}}(0)^{*}\right\rangle}{\left\langle\left|\varphi_{\mathbf{q}}\right|^{2}\right\rangle}$

We note that $S(\mathrm{q}, \mathrm{t})$ does not have to be exponential, as in the conventional $\mathrm{CHC}$ theory. The microscopic derivation does not contain phenomenological quantities such as the Onsager coefficient $\Lambda(q)$, nor does it require the incompressibility assumption.

In the nonlinear theory, one has to introduce approximations to express the third and fourth order correlation functions in Eq. 14 , in terms of $\mathrm{I}(\mathrm{q}, \mathrm{t})$. If we assume, following Langer (Ref.1,2), that $\varphi_{\mathbf{q}}(\mathrm{t})$ for different values of $\mathbf{q}$ are Gaussian random variables with zero mean, then we have $\left\langle\varphi_{\mathbf{q}_{1}}(\mathrm{t}) \varphi_{\mathbf{q}_{2}}{ }^{(t) \varphi_{-q}}{ }^{(t)}\right\rangle=0$ and

$$
\begin{aligned}
<\varphi_{\mathbf{q}_{1}}(\mathrm{t}) \varphi_{\mathbf{q}_{2}}(\mathrm{t}) \varphi_{\mathbf{q}_{3}}(\mathrm{t}) \varphi_{-} \mathbf{q}^{(\mathrm{t})>}= & <\varphi_{\mathbf{q}_{1}}(\mathrm{t}) \varphi_{\mathbf{q}_{2}}(\mathrm{t})><\varphi_{\mathbf{q}_{3}}(\mathrm{t}) \varphi_{-\mathbf{q}}(\mathrm{t})> \\
& +<\varphi_{\mathbf{q}_{1}}(\mathrm{t}) \varphi_{\mathbf{q}_{3}}(\mathrm{t})><\varphi_{\mathbf{q}_{2}}(\mathrm{t}) \varphi_{-\mathbf{q}}(\mathrm{t})> \\
& +<\varphi_{\mathbf{q}_{1}}(\mathrm{t}) \varphi_{-\mathbf{q}}(\mathrm{t})><\varphi_{\mathbf{q}_{2}}(\mathrm{t}) \varphi_{\mathbf{q}_{3}}(\mathrm{t})>
\end{aligned}
$$


Using $<\varphi \mathbf{q}_{i}{ }^{(t) \varphi} \mathbf{q}_{j}{ }^{(t)}>=<\varphi q_{i}{ }^{(t)} \varphi_{-q_{j}}{ }^{(t)}>\delta\left(\mathbf{q}_{i}+q_{j}\right)$, we find

$<\varphi_{\mathbf{q}_{1}}(\mathrm{t}) \varphi_{\mathbf{q}_{2}}(\mathrm{t}) \varphi_{\mathbf{q}_{3}}(\mathrm{t}) \varphi_{-} \mathbf{q}^{(\mathrm{t})}>=3\left(\frac{\mathrm{V}}{\mathrm{v}_{\mathrm{o}}}\right)^{2} \mathrm{I}(\mathrm{q}, \mathrm{t}) \mathrm{I}\left(\mathrm{q}_{1}, \mathrm{t}\right) \delta\left(\mathbf{q}_{1}-\mathbf{q}\right) \delta\left(\mathbf{q}_{2}+\mathbf{q}_{3}\right)$

Substitution of Eqs. 19 and 20 into Eq.14 yields

$\frac{\mathrm{dI}(\mathrm{q}, \mathrm{t})}{\mathrm{dt}}=-2 \mathrm{R}(\mathrm{q}) \mathrm{I}(\mathrm{q}, \mathrm{t})[1+\mathrm{Z}(\mathrm{q}, \mathrm{t})]+\mathrm{C}(\mathrm{q})$

where

$\mathrm{Z}(\mathrm{q}, \mathrm{t})=\frac{\mathrm{v}_{0}}{\mathrm{~V}} \sum_{\mathbf{q}^{\prime}} \gamma\left(\mathrm{q}, \mathrm{q}^{\prime}\right) I\left(\mathrm{q}^{\prime}, \mathrm{t}\right)$

In Eq. 22 we have introduced $\gamma\left(q, q^{\prime}\right)=3 \Gamma_{3}\left(\mathbf{q},-\mathbf{q}, \mathbf{q}^{\prime},-\mathbf{q}^{\prime}\right) \mathrm{S}_{\mathrm{AA}}(\mathrm{q})$ where $\Gamma_{3}\left(\mathbf{q}, \mathbf{q}_{1}, \mathbf{q}_{2}, \mathbf{q}_{3}\right)$ was defined above, and $\mathrm{S}_{\mathrm{AA}}(\mathrm{q})$ is given in $\mathrm{Eq} .2$. In the small-q limit, for example, one has

$$
\gamma\left(\mathrm{q}, \mathrm{q}^{\prime}\right)=\frac{3}{2} \frac{\mathrm{z}_{1}+\mathrm{Z}_{2}\left(\mathrm{q}^{2}+\mathrm{q}^{\prime 2}\right) / 3}{\chi_{\mathrm{s}}-\chi+\frac{\mathrm{q}^{2}}{36}\left[\frac{\sigma_{\mathrm{A}}^{2}}{\mathrm{z}_{\mathrm{A}} \phi_{0}}+\frac{\sigma_{\mathrm{B}}^{2}}{\mathrm{z}_{\mathrm{B}}\left(1-\phi_{0}\right)}\right]}
$$

where $Z_{1}$ and $Z_{2}$ are defined in Eqs.12a and 12b.

The assumption that the $\varphi_{\mathbf{q}}(\mathrm{t})$ for different values of $\mathbf{q}$ are Gausian random variables with zero mean, is equivalent to treating $\varphi(r, l)$ as Gaussian random variables with zero mean, at different values of $\mathbf{r}$, so that fourth order correlation functions in the configuration space can also be factorized. For example, $\left\langle\varphi^{3}(\mathbf{r}, \mathbf{t}) \varphi\left(\mathbf{r}^{\prime}, t\right)\right\rangle=3\left\langle\varphi^{2}(\mathbf{r}, \mathrm{t})\right\rangle\left\langle\varphi(\mathbf{r}, \mathbf{t}) \varphi\left(\mathbf{r}^{\prime}, \mathbf{t}\right)\right\rangle$, which was used by Langer (Ref.2). If $\gamma\left(q, q^{\prime}\right)$ in Eq.22 were independent of $q^{\prime}$ and equal to $\gamma(q)$, then the function $\mathrm{Z}(\mathrm{q}, \mathrm{t})$ could be excessed as the average variance of the local volume fraction fluctuations as

$$
Z(q, t)=\gamma(q) \frac{1}{V} \int_{V} d r<\varphi(r, t)^{2}>
$$

which follows from the Parseval relation relating the Fourier pairs. The Eq.22 shows that the function $Z(q, t)$ represents the effect of mode coupling on the time evolution of $I(q, t)$. Indeed, Eq. 21 can be solved in terms of $Z(q, t)$ as

$$
I(q, t)=I_{i n}(q) e^{-2 \int_{0}^{t} d t^{\prime} \Omega\left(q, t^{\prime}\right)}+C(q) \int_{0}^{t} d u e^{-2 \int_{u}^{t} d t^{\prime} \Omega\left(q, t^{\prime}\right)}
$$

where

$\Omega(\mathrm{q}, \mathrm{t})=\mathrm{R}(\mathrm{q})[1+\mathrm{Z}(\mathrm{q}, \mathrm{t})]$

which may be identified as an instantaneous relaxation frequency. In this sense, $Z(q, t)$ represents the relative contribution of mode coupling to the relaxation process, in addition to the relaxation frequency $R(q)$ in the linear theory. The magnitude of the noise source, $C(q)$, is determined from the requirement that $\mathrm{I}(\mathrm{q}, \mathrm{t} \rightarrow \infty)=\mathrm{I}_{\mathrm{eq}}(\mathrm{q})$, assuming again that the final state is in one-phase region : 
$C(q)=2 R(q) I_{e q}(q)\left[1+Z_{e q}(q)\right]$

where

$\mathrm{z}_{\mathrm{eq}}(\mathrm{q})=\frac{\mathrm{v}_{\mathrm{o}}}{\mathrm{V}} \sum_{\mathbf{q}^{\prime}} \gamma\left(\mathrm{q}, \mathrm{q}^{\prime}\right) \mathrm{I}_{\mathrm{eq}}\left(\mathrm{q}^{\prime}\right)$

In the linear theory $\mathrm{C}(\mathrm{q})$ was given by $2 \mathrm{R}(\mathrm{q}) \mathrm{I}_{\mathrm{eq}}(\mathrm{q})$ (cf. Eq.16). We find that in the nonlinear theory the magnitude of the Langevin random force is a nonlinear function of the static structure factor $I_{\mathrm{eq}}(\mathrm{q})$ in the final state, because of the additional factor $\left[1+Z_{\mathrm{eq}}(\mathrm{q})\right]$. The latter has not been included in the previous investigations reported in the literature (e.g., Ref.6,12). Eq.25 shows that the linear $\mathrm{CHC}$ form is recaptured when $\mathrm{Z}(\mathrm{q}, \mathrm{t})$ is set equal to zero.

\section{SCALED EQUATIONS}

It is convenient to rewrite Eq.21 in terms of dimensionless variables in order to bring about the universal aspects of the dissolution and demixing processes, by introducing the dimensionless wave number $k=q / q_{c}$, and the dimensionless time $\tau=q_{c}{ }^{2} D_{\text {int }} t$, where $q_{c}$ is the inverse correlation length $\zeta$, and $D_{\text {int }}$ is the interdiffusion coefficient. The $q_{c}$ is defined by expressing $S_{A A}(q)$ in Eq.5a as $S_{A A}(q)=S_{A A}(0) /\left[1+q^{2} / q_{c}^{2}\right)$ :

$\mathrm{q}_{\mathrm{c}}^{2}=\frac{3}{\mathrm{R}_{\mathrm{g}}^{2}}\left[1-\frac{\chi\left(\mathrm{T}_{\mathrm{f}}\right)}{\chi_{\mathrm{s}}}\right]$

where, and henceforth, we specialize to a completely symmetric mixture in which the chains of the components are identical: $\mathrm{N}_{\mathrm{A}}=\mathrm{N}_{\mathrm{B}}, \mathrm{z}_{\mathrm{A}}=\mathrm{z}_{\mathrm{B}}, \sigma_{\mathrm{A}}=\sigma_{\mathrm{B}}$, and $\mathrm{v}_{\mathrm{A}}=\mathrm{v}_{\mathrm{B}}$ and $\phi_{0}=1 / 2$. The critical interaction parameter is given by $\chi_{S}=2 / \mathrm{N}$ where $\mathrm{N}$ is the number of statistical segments in a chain. The interdiffusion coefficient defined as $D_{\text {int }}=\lim R(q) / q^{2}$ as $q \rightarrow 0$, i.e.,

$D_{\text {int }}=2 \Lambda\left(T_{f}\right)\left[\chi_{s}-\chi\left(T_{f}\right)\right]$

In these definitions, the final state is assumed to be in the one-phase region so that $\chi\left(T_{f}\right)<\chi_{s}$. The case of $\chi\left(T_{\mathrm{f}}\right)>\chi_{\mathrm{s}}$, corresponding to spinodal decomposition will be treated separately. In terms of the above dimensionless variables, Eq. 21 reads

$\frac{\mathrm{d}(\mathrm{k}, \tau)}{\mathrm{d} \tau}=-2 \mathrm{r}(\mathrm{k}) \mathrm{I}(\mathrm{k}, \tau)[1+\mathrm{Z}(\mathrm{k}, \tau)]+\mathrm{C}(\mathrm{k})$

where $r(k)=k^{2}\left(1+k^{2}\right)$, and $C(k)=2 r(k) I_{e q}(k)\left[1+Z_{e q}(k)\right]$, or explicitly

$\mathrm{C}(\mathrm{k})=\frac{\mathrm{k}^{2}}{\chi_{\mathrm{s}}-\chi\left(\mathrm{T}_{\mathrm{f}}\right)}\left[1+\mathrm{Z}_{\mathrm{eq}}(\mathrm{k})\right]$

$I_{c q}(k)=\frac{1}{2\left[\chi_{s}-\chi\left(T_{f}\right)\right]\left(1+k^{2}\right]}$ 
The calculation of $Z_{\text {eq }}(k)$ using Eq.27b requires some care. First, one must convert the discrete summation on $\mathbf{q}^{\prime}$ to an integration using $\Sigma_{\mathbf{q}^{\prime}} \rightarrow\left[\mathrm{V} /(2 \pi)^{3}\right] \int d \mathbf{q}^{\prime}$, and introduce an upper cut-off wavenumber $q_{\text {cut }}$. Then, Eq.27b reduces to

$Z_{e q}(q)=\frac{v_{0}}{2 \pi^{2}} \int_{0}^{q c u t} d q^{\prime} q^{\prime 2} \gamma\left(q, q^{\prime}\right) I_{e q}\left(q^{\prime}\right)$

where $\gamma\left(q, q^{\prime}\right)$ is defined in Eq.23, which reduces in the case of completely symmetric mixture to $\gamma\left(\mathrm{q}, \mathrm{q}^{\prime}\right)=\frac{4}{1-\chi\left(\mathrm{T}_{\mathrm{f}}\right) / \chi_{\mathrm{s}}} \frac{1+\left(\mathrm{q}^{2}+\mathrm{q}^{2}\right) \mathrm{R}_{\mathrm{q}}^{2} / 3}{1+\mathrm{q}^{2} / \mathrm{q}_{\mathrm{c}}^{2}}$

The reason for introducing a cut-off is that the integral in Eq.32 is divergent otherwise. The physical implication of $q_{c u t}$ is that only the modes with wavenumbers between zero and $q_{\text {cut }}$ are allowed to be coupled to each other. It is therefore reasonable to choose $\mathrm{q}_{\mathrm{cut}}$ at a value about the inverse of the correlation length $\zeta$ (Ref.2). The integration in Eq.32 can be performed analytically, after the substitution of Eqs. $31 \mathrm{~b}$ and 33 in its integrand, as

$$
\begin{aligned}
& \mathrm{Z}_{\mathrm{eq}}(\mathrm{k})=\frac{3 \sqrt{3}}{\pi^{2}} \frac{\mathrm{v}_{\mathrm{o}}}{\chi_{\mathrm{s}} \mathrm{R}_{\mathrm{g}}^{3}} \frac{1}{\sqrt{1-\chi\left(\mathrm{T}_{\mathrm{f}}\right) / \chi_{\mathrm{s}}}} \frac{1}{1+\mathrm{k}^{2}} \\
& (\mathrm{x})\left\{\left(1+\frac{\mathrm{R}_{\mathrm{g}}^{2} \mathrm{q}_{\mathrm{c}}^{2}\left(\mathrm{~T}_{\mathrm{f}}\right)}{3} \mathrm{k}^{2}\right)\left(\alpha-\tan ^{-1} \alpha\right)+\frac{\mathrm{R}_{\mathrm{g}}^{2} \mathrm{q}_{\mathrm{c}}^{2}\left(\mathrm{~T}_{\mathrm{f}}\right)}{3}\left(\tan ^{-1} \alpha+\frac{\alpha^{3}}{3}-\alpha\right)\right\}
\end{aligned}
$$

where we have introduced $\alpha=\mathrm{q}_{\mathrm{cut}} / \mathrm{q}_{\mathrm{c}}$ as a measure of the range of mode coupling. It is to be noted that $Z_{\mathrm{eq}}(\mathrm{k})$ diverges when the final state approaches the spinodal line.

The expression of $\mathrm{Z}(\mathrm{k}, \mathrm{t})$ in Eq. 30 can be written explicitly following the same procedure as in the calculation of $Z_{\text {eq }}(k)$ :

$\mathrm{Z}(\mathrm{k}, \tau)=\frac{6 \sqrt{3}}{\pi^{2}} \frac{\mathrm{v}_{\mathrm{o}}}{\mathrm{R}_{\mathrm{g}}^{3}} \frac{\sqrt{1-\chi\left(\mathrm{T}_{\mathrm{f}}\right) / \chi_{\mathrm{s}}}}{\left(1+\mathrm{k}^{2}\right)} \int_{0}^{\alpha} \mathrm{dx} \mathrm{x}^{2}\left[1+\frac{\mathrm{R}_{\mathrm{g}}^{2} \mathrm{q}_{\mathrm{c}}^{2}\left(\mathrm{~T}_{\mathrm{f}}\right)}{3}\left(\mathrm{k}^{2}+\mathrm{x}^{2}\right)\right] \mathrm{I}(\mathrm{x}, \tau)$

The input parameters to be specified to characterize a reverse quench experiment are $\chi_{S}$, $\chi\left(T_{f}\right), v_{0}$ and $R_{g}$. The $\alpha$ is an adjustable parameter in the theory with values in the vicinity of unity. In the numerical results presented below, it will be chosen to be unity. The initial intensity $I_{\text {in }}(k)=I(k, t=0)$ must also be specified either experimentally, or must be obtained by solving Eq. 21 in the case of a temperature jump from $T_{i}$ in the one phase region to $T_{f}$ in the spinodal region, and by allowing the system to phase separate through spinodal decomposition. Note that the temperature after a step change is always denoted by $T_{\mathrm{f}}$. Eq.35 shows that $Z(k, \tau)$ is positive at all times, thus indicating that the effect of the nonlinearity, and the mode coupling arising from it, always enhances the relaxation rate given in Eq.26. At large times, $Z(k, \tau) \rightarrow Z_{e q}(k)$, so that the relaxation frequency asymptotically approaches $\Omega(k)=r(k)\left[1+Z_{\mathrm{eq}}(k)\right]$. When the final state in the one-phase region is sufficiently away from 
the spinodal line, i.e., when $\chi\left(T_{f}\right)<<\chi_{S}$, the magnitude of $Z_{e q}(k)$ is small as compared to unity, so that the decay of the intensity follows the linear theory. Otherwise the linear theory is not valid even asymptotically.

In order to describe the spinodal decomposition analytically, the above equations are modified slightly because in this case $\chi\left(T_{f}\right)>\chi_{S}$, and the initial state is an equilibrium state at $T_{i}$ in onephase region. Since the final two-phase equilibrium state at $T_{f}$ is not accessible, the procedure followed above to determine $\mathrm{C}\left(\mathrm{q}, \mathrm{T}_{\mathrm{f}}\right)$, i.e., the magnitude of the random force after the the temperature jump, from the requirement $\mathrm{I}(\mathrm{q}, \mathrm{t} \rightarrow \infty)=\mathrm{I}_{\mathrm{eq}}(\mathrm{q})$ must also be modified. Since the homogeneous state of the blend immediately after the temperature jump to $T_{f}$ is an unstable one, the direct application of the fluctuation-dissipation theorem to determine $\mathrm{C}\left(\mathrm{q}, \mathrm{T}_{\mathrm{f}}\right)$ is questionable. This point was discussed by one of us in detail elsewhere (Ref.10). Here, we assume that the temperature difference $T_{f}-T_{i}$ is sufficiently small so that $C\left(q, T_{f}\right) \cong C\left(q, T_{i}\right)$. The latter is given Eq.27a in terms of the initial equilibrium intensity $\mathrm{I}_{\mathrm{eq}}\left(\mathrm{q}, \mathrm{T}_{\mathrm{i}}\right)$. With these modifications, we obtain the following equations in reduced variable for the description of spinodal decomposition:

$\frac{\mathrm{d} I(k, \tau)}{\mathrm{d} \tau}=2 \mathrm{r}(\mathrm{k}) \mathrm{I}(\mathrm{k}, \tau)[1+\mathrm{Z}(\mathrm{k}, \tau)]+\mathrm{C}\left(\mathbf{k}, \mathrm{T}_{\mathbf{f}}\right)$

where $r(k)=k^{2}\left(1-k^{2}\right)$ with $k=q / q_{c}\left(T_{f}\right)$, and

$\mathrm{Z}(\mathrm{k}, \tau)=-\frac{6 \sqrt{3}}{\pi^{2}} \frac{\mathrm{v}_{\mathrm{o}}}{\mathrm{R}_{\mathrm{g}}^{3}} \frac{\sqrt{\chi\left(\mathrm{T}_{\mathrm{f}}\right) / \chi_{\mathrm{s}}-1}}{\left(1-\mathrm{k}^{2}\right)} \int_{0}^{\alpha} \mathrm{dx} \mathrm{x}^{2}\left[1+\frac{\mathrm{R}_{\mathrm{g}}^{2} \mathrm{q}_{\mathrm{c}}^{2}\left(\mathrm{~T}_{\mathrm{f}}\right)}{3}\left(\mathrm{k}^{2}+\mathrm{x}^{2}\right)\right] \mathrm{I}(\mathrm{x}, \tau)$

where $\mathrm{q}_{\mathrm{c}}^{2}\left(\mathrm{~T}_{\mathrm{f}}\right)=\left(3 / \mathrm{R}_{\mathrm{g}}^{2}\right)\left[\chi\left(\mathrm{T}_{\mathrm{f}}\right) / \chi_{\mathrm{s}}-1\right]$. The interdiffusion coefficient is modified as $D_{\text {int }}=2 \Lambda\left(T_{f}\right)\left[\chi\left(T_{f}\right)-\chi_{s}\right]$. The initial intensity, which is the equilibrium intensity at the initial temperature $T_{i}$ is

$\mathbf{I}_{\text {in }}(\mathbf{k})=\frac{1}{2\left[\chi_{s}-\chi\left(T_{i}\right)\right]}\left[1+\frac{\chi\left(T_{f}\right)-\chi_{s}}{\chi_{s}-\chi\left(T_{i}\right)} k^{2}\right]^{-1}$

The factor in front of $\mathrm{k}^{2}$ in Eq. 38 arises from the fact that $\mathrm{k}$ denotes the normalized wavenumber relative to the correlation length at the final state. The magnitude of the fluctuations $C\left(k, T_{f}\right)$ in Eq.36 is calculated as

$\mathrm{C}\left(\mathrm{k}, \mathrm{T}_{\mathrm{f}}\right) \cong \frac{\mathrm{k}^{2}}{\chi\left(\mathrm{T}_{\mathrm{f}}\right)-\chi_{\mathrm{s}}}\left[1+\mathrm{Z}_{\mathrm{eq}}\left(\mathrm{k}, \mathrm{T}_{\mathrm{i}}\right)\right]$

where we have replaced the factor $\Lambda\left(T_{i}\right) / \Lambda\left(T_{f}\right)$ by unity assuming that the temperature jump $T_{f}-T_{i}$ is small, in order to reduce the number of parameters in the numerical calculations. The $\mathrm{Z}_{\mathrm{eq}}\left(\mathrm{k}, \mathrm{T}_{\mathbf{i}}\right)$ in $\mathrm{Eq} .39$ is given by 


$$
\begin{aligned}
\mathrm{Z}_{\mathrm{eq}}\left(\mathrm{k}, \mathrm{T}_{\mathrm{i}}\right)= & \frac{3 \sqrt{3}}{\pi^{2}} \frac{\mathrm{v}_{\mathrm{o}}}{\chi_{\mathrm{s}} \mathrm{R}_{\mathrm{g}}^{3}} \frac{1}{\sqrt{1-\chi\left(\mathrm{T}_{\mathrm{i}}\right) / \chi_{\mathrm{s}}}}\left[1+\frac{\chi\left(\mathrm{T}_{\mathrm{f}}\right)-\chi_{\mathrm{s}}}{\chi_{\mathrm{s}}-\chi\left(\mathrm{T}_{\mathrm{i}}\right)} \mathrm{k}^{2}\right]^{-1} \\
& (\mathrm{x})\left\{\left(1+\frac{\mathrm{R}_{\mathrm{g}}^{2} \mathrm{q}_{\mathrm{c}}^{2}\left(\mathrm{~T}_{\mathrm{i}}\right)}{3} \mathrm{k}^{2}\right)\left(\alpha-\tan ^{-1} \alpha\right)+\frac{\mathrm{R}_{\mathrm{g}}^{2} \mathrm{q}_{\mathrm{c}}^{2}\left(\mathrm{~T}_{\mathrm{i}}\right)}{3}\left(\tan ^{-1} \alpha+\frac{\alpha^{3}}{3}-\alpha\right)\right\}
\end{aligned}
$$

where $q_{c}^{2}\left(T_{i}\right)=\left(3 / R_{g}^{2}\right)\left[1-\chi\left(T_{i}\right) / \chi_{s}\right]$. It is to be noticed in Eq.37 that $r(k) Z(k, \tau)$ is always negative at any wavenumber, thus indicating that the nonlinearity slows down the growth rates, but enhances the decay rates.

The input parameters in the case of spinodal decomposition are: $\chi\left(\mathrm{T}_{\mathrm{i}}\right), \chi\left(\mathrm{T}_{\mathrm{f}}\right), \chi\left(\mathrm{T}_{\mathrm{S}}\right), \mathrm{v}_{\mathrm{O}}$ and $\mathrm{R}_{\mathrm{g}}$. Again, the parameter $\alpha=\left(\mathrm{q}_{\mathrm{cut}} / \mathrm{q}_{\mathrm{c}}\right)$ is to be adjusted around unity. In the following section, we present the variation of the intensity during a complete cycle starting from a temperature $T_{i}$, jumping to $T_{\Gamma}$ and allowing spinodal decomposition for a period of time, and then dropping it back to $T_{i}$.

\section{NUMERICAL RESULTS:}

\section{SPINODAL DECOMPOSITION}

The figures 1-4 are obtained by numerically solving Eq.36 with $\chi\left(\mathrm{T}_{\mathrm{i}}\right)=0.95 \chi_{\mathrm{s}}, \chi\left(\mathrm{T}_{\mathrm{f}}\right)=1.10$ $\chi_{\mathrm{S}}, \chi_{\mathrm{s}}=9.024 \times 10^{-4}, v_{\mathrm{o}}=0.922 \times 10^{-20} \mathrm{~cm}^{3}, \alpha=1$ and $\mathrm{R}_{\mathrm{g}}{ }^{2}=6.25 \times 10^{-12} \mathrm{~cm}^{2}$. The curve at $\tau=0$ in all these curves denotes the equilibrium intensity in the initial state. Figure 1 shows the variation of intensity at different times during spinodal decomposition in the presence of mode coupling. The shift of the location of the peak towards smaller values of wavenumber is clearly observable.

Fig. 2 is plotted using the linear $\mathrm{CHC}$ theory for comparison with the nonlinear theory. It is seen that the linear theory predicts a very rapid growth even at short times. The mode coupling arising from the nonlinearity decreases the growth rates, but enhances the decay rates.

Figure 3 shows that the location of the peak intensity decreases as a function of time, and follows asymptotically a power law as $\ln \mathrm{k}_{\mathrm{m}}(\tau)=-0.18-0.25 \ln \tau$ for large times. Although this prediction is in good agreement with the asymptotic $\mathrm{t}^{-0.27}$-power law behavior observed in the recent experiments by Han (Ref. 14) and his coworkers, it does not represent the true asymptotic behavior which we obtained analytically as $\ln \mathrm{k}_{\mathrm{m}}(\tau)=-0.219-(1 / 3) \ln \tau$. It seems that the exact $t^{-1 / 3}$-power law behavior is not reached in the time interval up to $\tau=128$. Later numerical calculations extended up to $\tau=512$ resulted in an exponent 0.32 , verifying the analytical prediction. However, this asymptotic behavior is restricted to the early stages of the spinodal decomposition. At the final stages, where the hydrodynamic effects play a dominant role, a power law as $\mathbf{t}^{-1}$ is expected (Ref.15).

The analytical derivation also shows, as predicted in Ref.16, that the intensity is scaled as $I(k, \tau)=k_{m}(\tau)^{-3} f\left[k / k_{m}(\tau)\right]$. The function $f(x)$ is interpolated between its small and large argument behavior found analytically to yield $f(x)=f(1) 3 x^{2} /\left(2+x^{6}\right)$ such that its maximum 

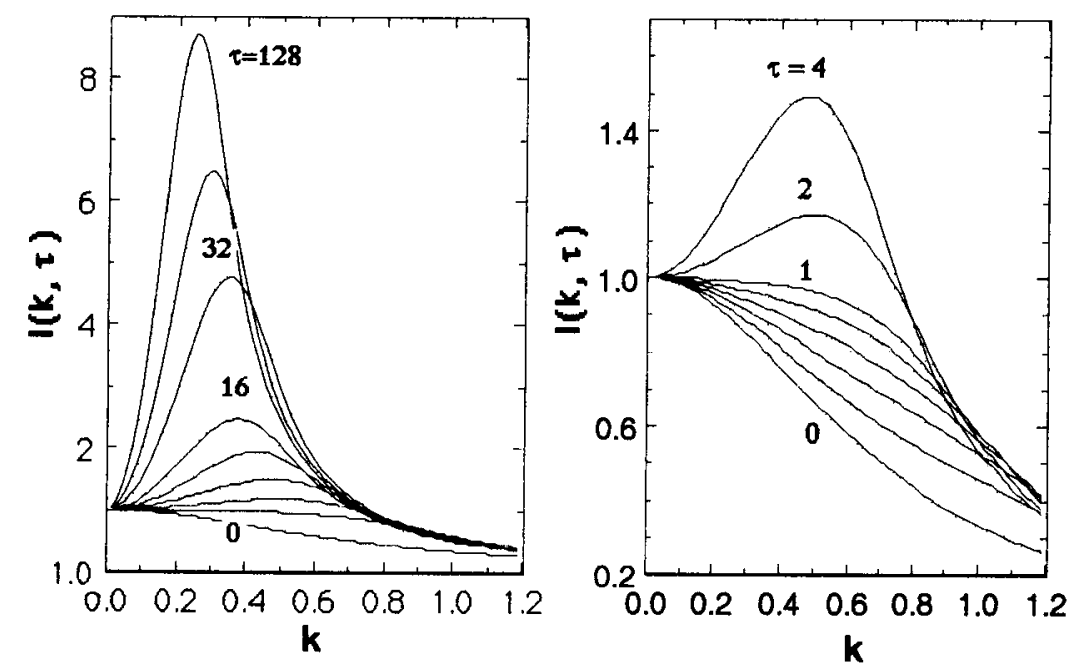

Fig.1. Variation of the intensily during spinodal decomposition with the normalized wavenumber $\mathrm{k}$ for the values of normalized time $\tau=0,0.2,0.4,0.6,0.8,1,2$, $4,8,16,32,64$, and 128 with mode coupling.

occurs at $x=1$. This form was suggested by Furukawa (Ref.17) some time ago.The value $f(1)$ is also calculated explicitly , and found to depend on temperature. These analytical derivation will be represented in a future publication.

Figure 4 shows the variation of the normalized peak intensity $\mathrm{I}\left(\mathrm{k}_{\mathrm{m}}(\tau), \tau\right) / \mathrm{I}_{\mathrm{eq}}(0)$ as a function of the normalized time. For large times it also obeys a power law as $\tau^{1 / 3}$. Figure 5 depicts the variation of the instantaneous relaxation frequency $\Omega(\mathrm{k}, \tau)=\mathrm{r}(\mathrm{k})[1+Z(\mathrm{k}, \tau)]$ as a function of $\mathrm{k}^{2}$ at different times $\tau$. In the linear theory, $Z(k, \tau)=0$, and $\Omega(k, \tau) / \mathrm{k}^{2}=1-\mathrm{k}^{2}$, which is independent of time (The top straight line in the figure). Since $Z(k, \tau)$ depends on both $k$ and $\tau$ in the nonlinear theory, the slope as well as the intercept of $\Omega(\mathrm{k}, \tau) / \mathrm{k}^{2}$ changes with time. If $\left(1-\mathrm{k}^{2}\right) \mathrm{Z}(\mathrm{k}, \tau)$ were independent of wavenumber, but dependent on time only, as it is the case in the Langer's original theory for fluids, then $\Omega(\mathrm{k}, \tau) / \mathrm{k}^{2}$ would be simply shifted downwards with the same slope as in the linear theory. Fig.5 shows however that there is a slight change with time in the slope. This additional $k$-dependence of $\Omega(k, \tau) / \mathrm{k}^{2}$ arises from the chain connectivity in polymer blends, which is accounted for by the term proportional to $\mathrm{R}_{\mathrm{g}}{ }^{2}$ in the integrand of the expression of $Z(k, \tau)$ in Eq.37. Another feature to be observed in Fig.5 is the variation with time of the intercept of $\Omega(\mathrm{k}, \tau) / \mathrm{k}^{2}$ with the $\mathrm{y}$-axis. The intercept is given by $[1+Z(0, \tau)]$, in which $Z(0, \tau)$ is always negative (see Eq.37). The initial value of $Z(0,0)$ is calculated by replacing $I(k, \tau)$ in $E q .37$ by $I_{\text {in }}(k)$ given by $E q .38$. The latter shows that the magnitude of $\mathrm{I}_{\mathrm{in}}(\mathrm{k})$ increases when the initial equilibrium state at $\mathrm{T}_{\mathrm{i}}$ approaches the spinodal line, i.e., when $\chi\left(T_{i}\right) \rightarrow \chi_{S}$. With the value $\chi\left(T_{j}\right)=0.95 \chi_{S}$, Eq.37 yields $Z(0,0) \cong-0.53$ so that $[1+Z(0,0)]=0.47$ (See Fig.5). The implication of this result is that the linear theory is not valid 

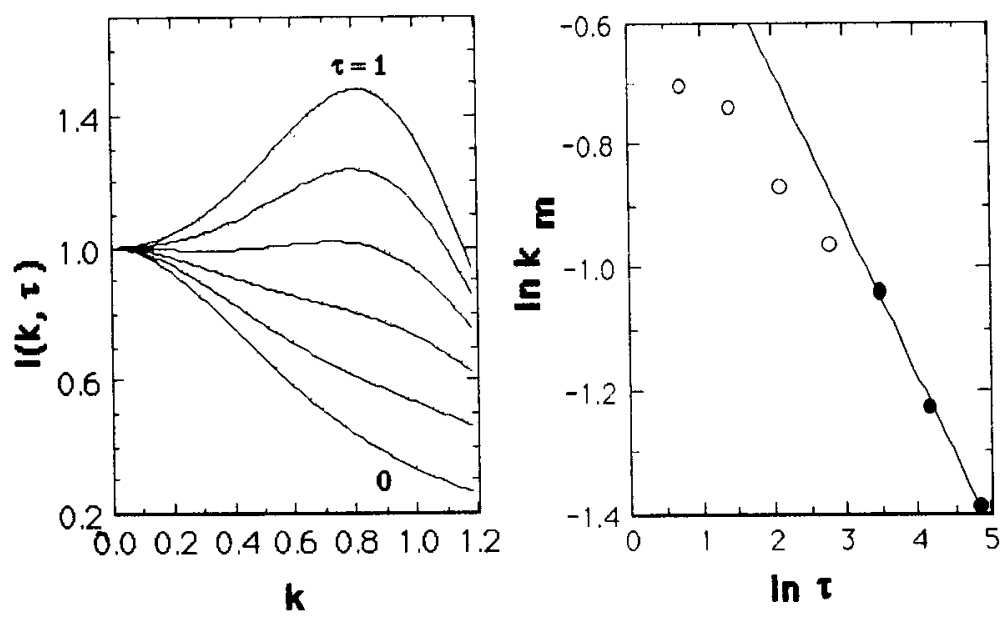

Fig.2. Variation of the intensity with the normalized wavenumber $\mathrm{k}$ during spinodal decomposition for different values of the normalized time $\tau=0.2,0.4,0.6,0.8$, I with the linear theory.

Fig.3. Variation of the localion of the peak intensity, $\mathrm{k}_{\mathrm{m}}(\tau)$, as a function of time.

even at short times when the initial state is close to the spinodal line. When it is far from the latter, then $|Z(0, \tau)|<<1$ and the linear theory is valid at the very early stages. Finally, Fig.5 shows that the q-range of the unstable modes for which $\Omega(k, \tau)>0$, is determined by $1+Z(k, \tau)>0$, and decreases with time. In the linear theory, all the modes for which $k<1$ grow in time.

\section{DISSOLUTION}

Figures 6,7 and 8 represent a reverse quench experiment in which the temperature is dropped from $\chi\left(T_{\mathfrak{f}}\right)=1.1 \chi_{\mathrm{S}}$ to $\chi\left(\mathrm{T}_{\mathrm{j}}\right)=0.95 \chi_{\mathrm{S}}$ at $\tau=128$ during the demixing processes. The top curve in Fig. 6 corresponds to the intensity $I(k, \tau=128)$ in Fig. 1 arrived at during the spinodal decomposition, which is taken to be the initial intensity in the reverse quench experiment. The lowest curve in Fig. 6 is the equilibrium intensity at $\chi\left(T_{i}\right)=0.95 \chi_{\mathbf{s}}$, which was the initial state in the spinodal decomposition. The intermediate curves in Fig.6 represent the change in the intensity during the dissolution process at $\tau=0.2,0.4,0.8,2,4$ and 8 in descending order. We note that $\mathrm{k}$ in Fig.6 is still normalized as $\mathrm{k}=\mathrm{q} / \mathrm{q}_{\mathrm{c}}\left(\mathrm{T}_{\mathrm{f}}\right)$, i.e., the same normalization used in Figs.1-4. Figure 7 shows the variation of the location of the peak intensity as a function of time. It is observed that $\mathrm{k}_{\mathrm{m}}(\tau)$ behaves asymptotically as $\tau^{-1 / 2}$. This behavior, which has also been observed experimentally (Ref.14), can be explained theoretically by differentiating Eq.25 with respect to $\mathrm{q}$ (more conveniently with respect to $\mathrm{q}^{2}$ ) and equating the result to zero. (It is more convenient not to use normalized variables in this derivation). The power-law behaviour occures in the early stages of dissolution in which the effect of thermal fluctuations represented in Eq. 25 by the second term is not yet important. Ignoring this term we find 

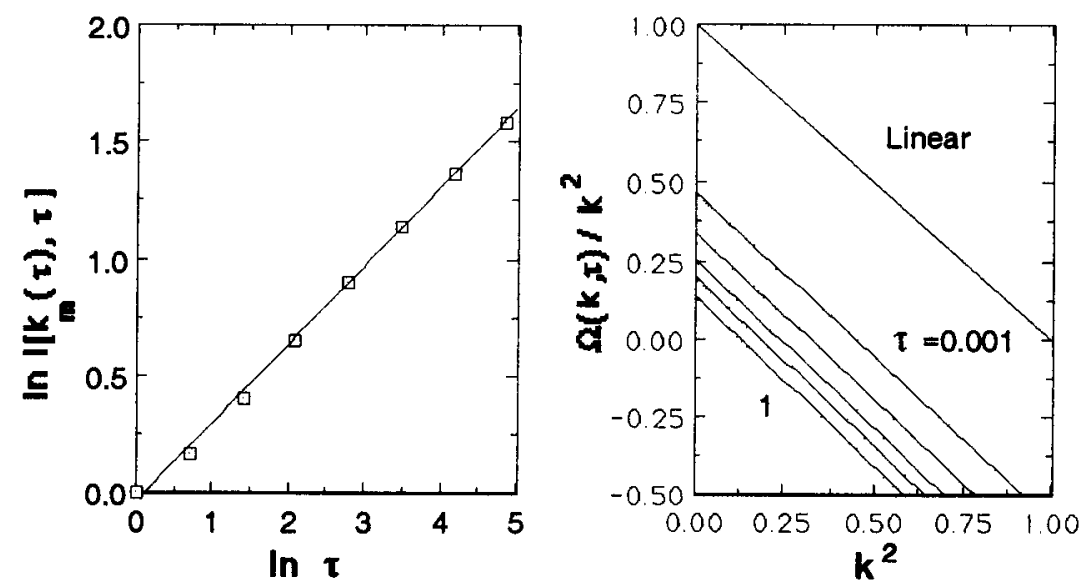

Fig.4. Variation of the normalized peak intensity as a function of time.

Fig.5. Variation of the relaxation frequency with wavenumber at different times

$$
\frac{d \ln I_{i n}(q)}{d q^{2}} i_{q=q_{m}(t)}=2 \frac{d}{d q^{2}}\left\{R(q)\left[t+\int_{o}^{t} d t^{\prime} Z\left(q, t^{\prime}\right)\right]\right\}_{q=q_{m}(t)}
$$

We evaluate this equation in the limit of $t \rightarrow \infty$ and $\mathrm{q}_{\mathrm{m}}(\tau) \rightarrow 0$. Using the fact that $\mathrm{Z}(\mathrm{q}, \mathrm{t} \rightarrow \infty)=\mathrm{Z}_{\mathrm{eq}}(\mathrm{q})$ for large times, we obtain

$$
\left.\frac{\mathrm{d} \ln \mathrm{I}_{\text {in }}(\mathrm{q})}{\mathrm{dq}^{2}}\right|_{\mathrm{q}=\mathrm{q}_{\mathrm{m}}(\mathrm{t})}=\left.2 \mathrm{t}\left[1+\mathrm{Z}_{\mathrm{eq}}(0)\right] \frac{\mathrm{dR}(\mathrm{q})}{\mathrm{dq}^{2}}\right|_{\mathrm{q}=\mathrm{q}_{\mathrm{m}}(\mathrm{t})}
$$

where $Z_{\mathrm{eq}}(0)$ follows from $\mathrm{Eq} .40$ as

$$
\mathrm{Z}_{\mathrm{eq}}(0)=\frac{3 \sqrt{3}}{\pi^{2}} \frac{\mathrm{v}_{0}}{\chi_{\mathrm{s}} \mathrm{R}_{\mathrm{g}}^{3}} \frac{1}{\sqrt{1-\chi\left(\mathrm{T}_{\mathrm{i}}\right) / \chi_{\mathrm{s}}}}\left\{\left(\alpha-\tan ^{-1} \alpha\right)+\frac{\mathrm{R}_{\mathrm{g}}^{2} \mathrm{q}_{\mathrm{c}}^{2}\left(\mathrm{~T}_{\mathrm{j}}\right)}{3}\left(\tan ^{-1} \alpha+\frac{\alpha^{3}}{3}-\alpha\right)\right\}
$$

which is small as compared to unity unless $\chi\left(\mathrm{T}_{\mathrm{j}}\right)$ is very close $\chi_{\mathrm{s}}$. The crucial quantity in Eq.41 is the variation of $\ln I_{\text {in }}(q)$ with $q$, which depends on the preparation of the initial state. In the reverse quench experiments we are interested in, the latter is prepared by spinodal decomposition starting from an equilibrium state, and by allowing the system to phase-separate sufficiently in the time interval $\mathrm{t}_{\mathrm{W}}$, so that the intermediate stage is reached. In this stage of spinodal decomposition, the intensity obeys dynamic scaling (Ref. 16, 17) such that its qdependence at a time $t$, in particular at $t_{W}$, can be represented as $I_{i n}(q)-q_{m}\left(t_{w}\right)^{-3} x^{2} /\left(2+x^{6}\right)$ where $x=q / q_{m}\left(t_{w}\right)$. Therefore, $d \ln I_{i n}(q) / d q^{2} I_{q=q_{m}}(t)=q_{m}(t)^{-2}$ in the limit of $q_{m}(t) \rightarrow 0$. 

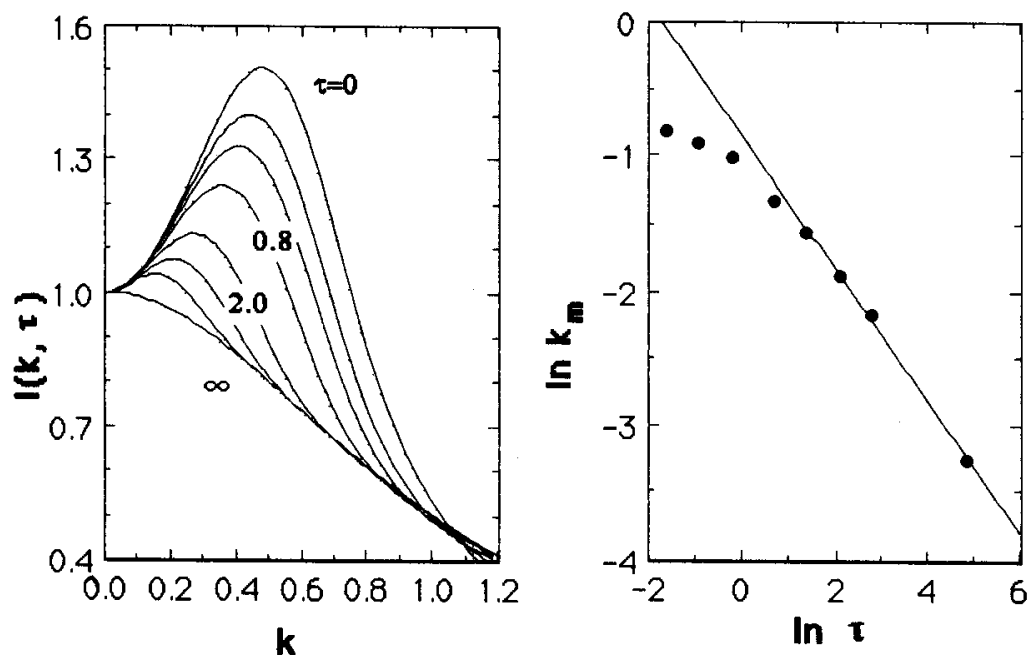

Fig.6. Variation of the intensity with the normalized wavenumber $\mathrm{k}$ for the values of normalized time $\tau=0.2,0.4,0.8,2,4$ and 8 in descending order, following a temperature drop from spinodal into one-phase region (reverse quench).

Fig.7. Variation of the location of the peak intensity $\mathrm{k}_{\mathrm{m}}(\tau)$ as a function of time after reverse quench

Substitution of this result and $d R(q) / d q^{2}=2 \Lambda\left(T_{i}\right)\left[\chi_{s}-\chi\left(T_{i}\right)\right]=D_{\text {int }}\left(T_{i}\right)$ into Eq.41 yields $\mathrm{q}_{\mathrm{m}}(\mathrm{t})^{2} \rightarrow \frac{1}{2\left[1+\mathrm{Z}_{\mathrm{eq}}(0)\right] \mathrm{D}_{\mathrm{int}} \mathrm{t}}$

which explains the observed asymptotic $\mathrm{t}^{-0.5}$-dependence in Fig.7. It also shows that the slope of the asymptote is related to the interdiffusion coefficient at the final temperature, thereby suggesting a method of inferring $D_{\text {int }}\left(T_{i}\right)$ from the $q_{m}(t)$ vs. $t$-data in a reverse quench experiment. In Fig.7, $q_{m}(t)$ is normalized as $k_{m}(\tau)=q_{m}(t) / q_{c}\left(T_{f}\right)$ and the time is normalized as $\tau=D_{\text {int }}\left(T_{i}\right) q_{c}\left(T_{i}\right)^{2}$. Using $q_{c}\left(T_{f}\right)^{2}=2 q_{c}\left(T_{i}\right)^{2}$ when $\chi\left(T_{i}\right)=0.95 \chi_{s}$ and $\chi_{\left(T_{f}\right)=1.1} \chi_{s}$, we find $\mathrm{k}_{\mathrm{m}}(\tau)^{2}=1 / 4\left[1+\mathrm{Z}_{\mathrm{eq}}(0)\right] \tau$. The numerical value of $\mathrm{Z}_{\mathrm{eq}}(0)$ is obtained from Eq.42 as 0.48 , so that $1 n k_{m}(\tau)=-0.89-0.5 \ln \tau$. The latter agrees well with the slope in Fig. 7 which is obtained by numerical integration as $1 \operatorname{lnk}_{\mathrm{m}}(\tau)=-0.85-0.5 \ln \tau$. It is important to note that the time dependence of $k_{m}(\tau)$ is determined by the behavior of the initial intensity as a function of $q$ in the small- $q$ region. Had the initial intensity been prepared differently, instead of by spinodal decomposition, then $\mathrm{k}_{\mathrm{m}}(\tau)$ could conceivably display a different time dependence. The variation of the peak intensity $I\left[\mathrm{k}_{\mathrm{m}}(\tau), \tau\right]$ with time following a reverse quench is represented in Figure 8.

\section{STEP TEMPERATURE CHANGES IN ONE-PIIASE REGION}

Figure 9 shows the variation of the intensity following a temperature drop involving a change in the interaction parameter from $\chi_{\mathrm{i}}=0.9 \chi_{\mathrm{s}}$ to $\chi_{\mathrm{f}}=0.1 \chi_{\mathrm{s}}$. The behavior of $\mathrm{I}(\mathrm{k}, \tau)$ does not 

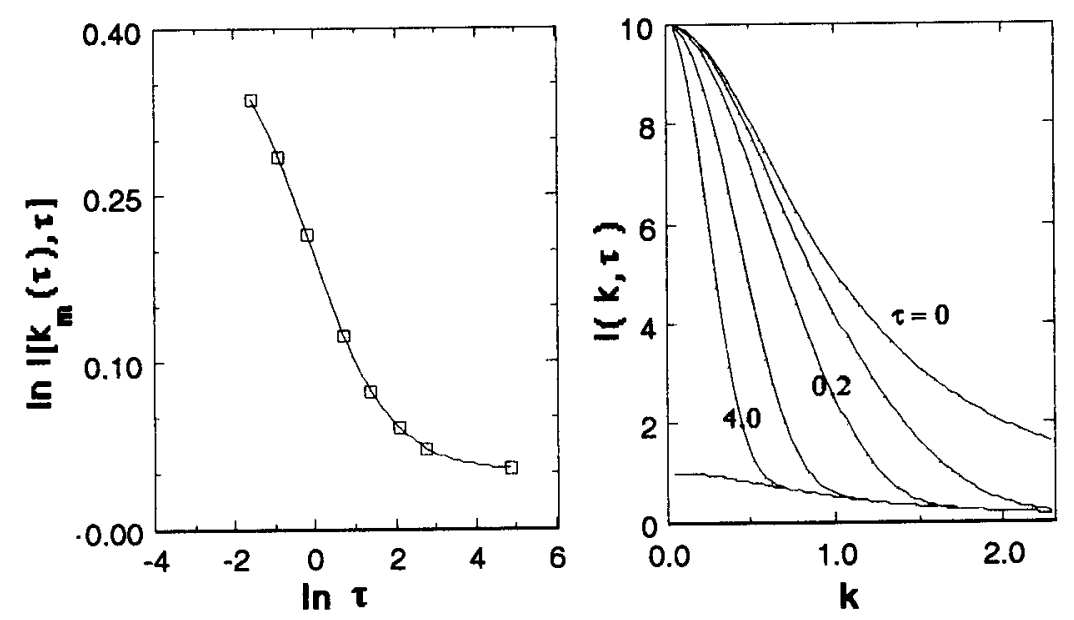

Fig.8. Variation of the peak intensity with time following a reverse quench.

Fig.9. Variation of the intensity following a temperature drop within one-phase region.

display any interesting structure: It decreases from its initial to its final equilibrium value monotonically. Since the long wavelengths do not decay, $I(k, \tau)$ display a peak at $q=0$ at all times. The lincar and nonlinear theories yield almost identical results, when the initial temperature is not too close to the spinodal temperature. The mode coupling tends to increase the relaxation rates. Its effect would be more noticeable during the early relaxation times, if the initial interaction parameter $\chi_{\mathrm{i}} / \chi_{\mathrm{s}}$ were closer to unity than 0.9 .

The variation of the intensity following a temperature jump from $\chi_{\mathrm{i}}=0.1 \chi_{\mathrm{s}}$ to $\chi_{1}=0.9 \chi_{\mathrm{s}}$ is presented in Fig. 10. The appearance of a peak, similar to the case of spinodal decomposition, is somewhat surprizing because there is no coarsening during the transition from an equilibrium state at a lower temperature to one at a higher temperature. The change in the intensity reflects the increase both in the magnitude of thermal fluctuations and the correlation length. The latter indicates an increase in the size of statistically correlated domains, and thus may be interpreted as " coarsening" in a statistical sense. The variation of the location of the peak as function of time is ploted in Fig. 11. Assuming that $I\left[\mathrm{k}_{\mathrm{m}}(\tau), \tau\right]$ saturates to $\mathrm{I}_{\mathrm{eq}}\left[\mathrm{k}_{\mathrm{m}}(\tau)\right]$ at the final temperature, we could show that $\mathrm{k}_{\mathrm{m}}(\tau)$ follows a power law as $\mathrm{t}^{-1 / 3}$ as found numerically. The proportionality constant, however, is not a universal number as it was the case in spinodal decomposition, and depends on the initial and final equilibrium intensities.In the case of a temperature jump also, the linear and nonlinear theories give very similar results when the final interaction parameter is lar below its spinodal value $\chi_{s}$. Otherwise, the effect of mode coupling hecomes appreciable, causing slowing down of the relaxation process. 

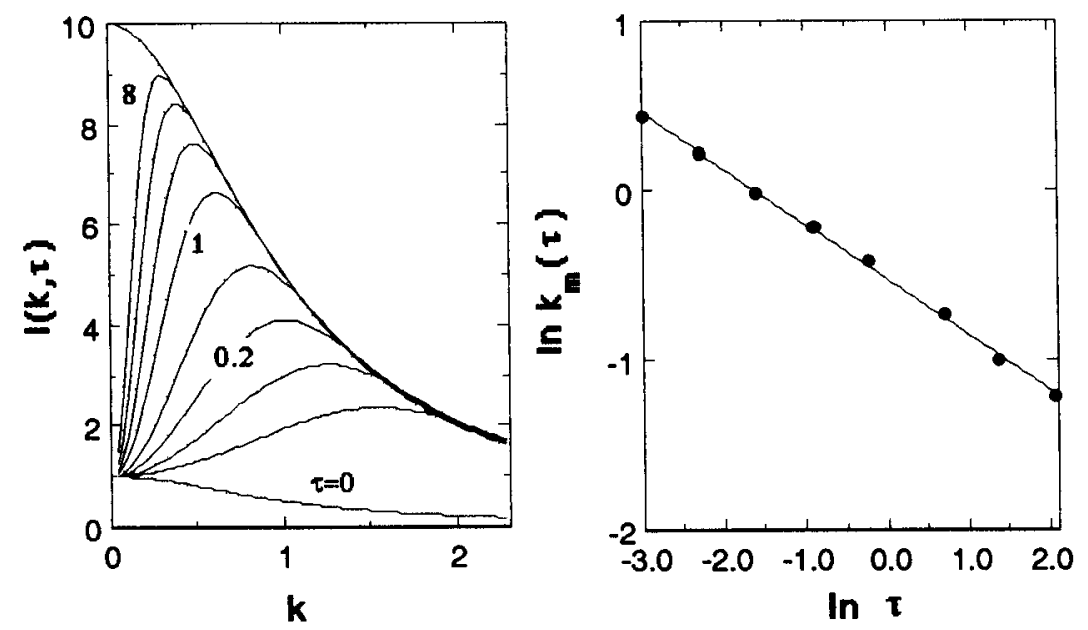

Fig. 10. Variation of the intensity following a temperature jump in one-phase region Fig.11. Variation of the location of the peak intensity $\mathrm{k}_{\mathrm{m}}(\tau)$ with time after a temperature jump in one-phase region.

\section{CONCLUSIONS}

The distinctive aspects of the theoretical approach used in this study are: a)The derivations are carried out directly in the q-space, and a closed equation for the measured intensity is obtained by treating the nonlinearity in the lowest order in the order parameter, and introducing Gaussian approximation. b) The polymer effects arising from chain connectivity are included in the calculations. c) The effect of thermal fluctuations is formulated differently from the conventional theories, by modifying the fluctuation-dissipation theorem in the presence of nonlinearities. d) The theoretical model is used to study the transients in intensity not only during spinodal decomposition, but also the transients following a reverse quench (dissolution of homogeneities) and step temperature changes in one phase region. The main qualitative conclusions are: a) The linear theory is not valid when the homogeneous initial equilibrium state is very close to the critical point, even at the very early stages of spinodal decomposition, because of the effect of mode-coupling on the growth rate. b) The linear behavior is not approached, as one would expect, even asymptotically at the later stages of dissolution following a reverse quench when the final equilibrium state in the one-phase region is very close to the spinodal line for the same reason as above. c) The effect of mode-coupling on the relaxation frequency may be interpreted as a renormalization of the Onsager coefficient (Ref. 18$)$ as $\Lambda_{\mathrm{nom}}(\mathrm{q}, \mathrm{l})=\Lambda[1+\mathrm{Z}(\mathrm{q}, \mathrm{t})]$ in $\mathrm{Eq} .21$. d) The model predicts asymptotic power-law dependence of the characteristic length $\mathrm{qm}_{\mathrm{m}}(\mathrm{t})^{-1}$ as a function of time both in spinodal decomposition and dissolution processes. The exponents has been found in good agreement with the recent experiments by C. S. Han, and his collaborators (Ref.14). 
ACKNOWLEDGEMENT: One of us (A. Z. A.) thanks the Ankara office of the United Nations for providing financial support under the program TOKTEN for his visit to Bogazici University during May, 1991.

\section{REFERENCES}

(1) J. S. Langer, M. Bar-on, H.D. Miller, Phys, Rev. A 11, 1417 (1975)

(2) J. S. Langer, 'Nonlinear Phenomena at Phase Transitions and Instabilities' (Nato Advanced Study Institute, 1981, Geilo, Norway) Edt.by T. Riste, Plenum Press, New York (1981)

(3) C. C. Han, M. Okada, Y. Muroga, F. L. McCrackin, B. J. Bauer, Q. Tran-Cong, Polym. Engineering and Sci. 26, 3 (1986).

(4) M. Sato and C.C. Han, J. Chem. Phys. 88, 1 (1988)

(5) P. G. de Gennes, 'Scaling Concepts in Polymer Physics', Cornell Univ.Press, Ithaca, (1979)

(6) K. Binder, J. Chem. Phys. 79, 6387 (1983)

(7) M. Shibayama, H. Yang, R. S. Stein, Macromolecules 18, 2179 (1985)

(8) P. G. de Gennes, J. Chem Phys. 72, 4756 (1980)

(9) A. Z. Akcasu, I. C. Sanchez, J. Chem Phys. 88, 7847 (1988)

(10) A. Z. Akcasu, Macromolecules 22, 3682 (1989)

(11) J.W.Cahn and J.E. Hilliard, J. Chem. Phys, 28, 258 (1958)

(12) H. E. Cook, Acta Metallurgica 18, 297 (1970)

(13) M. Lax, Rev.Mod. Phys. 32, 25 ( 1960)

(14) Private communication.

(15) A. Chakrabarti, R. Toral, J. D. Gunton, M. Muthukumar, L. Chem. Phys. 92, 6899 (1990)

(16) K. Binder and D. Stauffer, Phys. Rev, lett., 33, 1006 (1974)

(17) H. Furukawa, Physica A. 123, 497 (1984)

(18) G. H. Fredricson, J. Chem. Phys. 85(6), 3556 (1986) 